\title{
Efecto del Tipo de Labranza con Tracción Animal en las Características Físicas del Suelo, Conservación de la Humedad y en el Crecimiento y Producción del Cultivo de la Papa
}

\author{
Pablo Mamani ${ }^{1}$, Rubén Botello ${ }^{1}$, Bruno Condori ${ }^{1}$, Humberto \\ Moya $^{2}$, André Devaux ${ }^{3}$
}

\begin{abstract}
Resumen
El estudio se realizó durante la campaña 1995-96 en la Estación Experimental Toralapa en Bolivia, ubicada a 3,430 m de altitud. Se establecieron dos ensayos, uno sobre las épocas y número de labranzas usando el arado de palo, y el otro evaluando implementos de labranza que comparaban el arado de palo (tradicional) con mejorados, arados reversible y de cincel, todos con tracción animal. En ambos ensayos se buscó determinar el efecto de los tratamientos en la conservación de la humedad en el suelo, en las propiedades físicas del suelo, en la calidad y eficiencia de la labranza y en la producción del cultivo de papa. La humedad del suelo fue evaluada con el método gravimétrico, las propiedades del suelo con el método del "perfil edafológico" y del "perfil cultural", la calidad de las labranzas con observaciones de la superficie arada.
\end{abstract}

La labranza antes de la siembra permite conservar más agua en el suelo en comparación con un suelo sin labranza, debido a que ésta logra romper los microporos de la capa arable. Las condiciones de alta precipitación de la campaña (20\% más respecto al promedio histórico) no permitió demostrar claramente las bondades del arado de cincel como acopiador de aguas. El porcentaje de desterronamiento se incrementa cuando las aradas se realizan con más anticipación a la siembra, debido a que las condiciones climáticas y la humedad del suelo favorecen este efecto. El arado reversible logra una mayor incorporación de rastrojo y logra una mayor superficie removida respecto al arado de palo y al arado de

Investigador Fundación PROINPA. Casilla 4285. Cochabamba, Bolivia.

Asistente Técnico Fundación PROINPA. Casilla 4285. Cochabamba, Bolivia.

Investigador CIP/ Proyecto Papa Andina. Apartado 1558. Lima, Perú. 
Cincel. La rayada y cruzada con arado de cincel demanda más tiempo respecto al arado de palo y al arado reversible. Con tres aradas, el arado de cincel permite una mayor profundidad de aradura respecto al arado reversible y al arado de palo, llegando a raspar el pie de arado y a romperlo en algunos sectores, permitiendo así un enraizamiento más uniforme. Un número de aradas mayor a tres con arado de palo, no repercute en un incremento del rendimiento. Finalmente, no se observaron diferencias significativas en el rendimiento de la papa por efecto de los implementos evaluados a excepción del uso de rastra de aletas.

\section{Palabras claves adicionales: Labranza, implementos, Características físicas del suelo. \\ Effect of Tillage Method with Animal Traction on Physical Soil Characteristics, Soil Moisture Conservation, and Growth and Production of Potato Crop}

\section{Summary}

This study was carried out during 1995-96 season in the Experimental Station Toralapa in Bolivia, located at an altitude of $3,430 \mathrm{~m}$. Two trials were established, one on tillage timing and number of passes using the traditional plough: "Arado de palo" and a second trial comparing the traditional plough with reversible and chisel ploughs. The objectives of both trials were to determine the effect of treatments on the conservation of moisture in the soil, on the soil physical properties, on tillage efficiency and on potato yields. Soil moisture was evaluated by the gravimetric method and soil properties evaluated using two approaches: the "soil profile" method; and, "tillage profile" method.

Tillage before planting allows conserving soil moisture when compared to soil without tillage, because tillage breaks the micropores in the cultivated soil layer. However, the high precipitation conditions during this season ( $20 \%$ above the historical average), did not allow observing clearly the advantage of the chisel plow as a water "harvester". The percentage of clods was reduced when tillage is applied early in the season because soil moisture was higher. 
The reversible plough contributes to a better straw incorporation in the soil and a greater soil turn over as compared to the traditional plough and the chisel plough. Tilling with the chisel plough demands more time as compared to the other two types of ploughs. The chisel plough also allows a deeper tilling with better and more uniform plant rooting. It appears that tilling more than 3 times does not result in an increase of yield. Finally, under the conditions of the trial, the type of ploughs evaluated did not show a significant effect on potato production except for the use of an improved rake.

Additional Index words: Tillage, implements, soil physical, characteristics.

\section{Introducción}

La diversidad geomorfológica y climática de la región andina da lugar a la existencia de distintos sistemas de producción, con diferentes capacidades productivas, destacando aquellos de baja productividad cuyas causas principales son: la baja calidad de los suelos (bajo contenido de materia orgánica) y el alto riesgo de ocurrencia de sequías.

El promedio histórico de precipitación en sistemas de alto riesgo de sequía de La Paz, Potosí, Chuquisaca y Cochabamba, difícilmente sobrepasa los $450 \mathrm{~mm} / a n ̃ o$. En algunas comunidades, si bien la cantidad total puede ser aceptable para la producción de cultivos, su mala distribución hace que existan períodos de sequía y períodos con fuertes lluvias lo que repercute en la baja productividad de los cultivos y en la erosión de los suelos.

Según Orsag (8), la labranza constituye una alternativa importante para regular la humedad en el suelo, aspecto que le impulsó a estudiar los regímenes hídricos del suelo del altiplano por efecto de la labranza. Herve (5) al referirse a la labranza señala que las preguntas que se hacen los agricultores con la finalidad de producir más o de asegurar una producción al menor costo son: ¿Cómo y cuándo labrar?, ¿Qué riesgos de erosión acarrea cada técnica de labranza?, ¿En qué estado quedan los suelos luego de estas intervenciones?, ¿En qué condiciones se puede instalar un cultivo sin labrar o con labranza mínima?, ¿Cuáles son las consecuencias sobre los cultivos?, etc. 
PROINPA (9) señala que en las zonas andinas, las operaciones de labranza están sujetas a la disponibilidad de humedad en el suelo. Labranzas en épocas no adecuadas conducen a inoportunos y excesivos movimientos de tierra, que si bien a corto plazo podrían mejorar la producción del cultivo de papa, con el tiempo repercuten en la degradación de los suelos, principalmente en parcelas en laderas.

Estos antecedentes motivaron a realizar el presente estudio, cuyo objetivo fue determinar el efecto del momento y del número de pases con arado de palo. Además definir las ventajas de implementos de labranza mejorados sobre la conservación de humedad en el suelo, las propiedades físicas del suelo, la calidad de labranza, el crecimiento y la producción del cultivo de papa.

\section{Materiales y Métodos}

La investigación se llevó a cabo en la campaña agrícola 1995 - 96, en la Estación Experimental Toralapa, ubicada en la provincia Tiraque del departamento de Cochabamba (Bolivia), a una altitud de 3,430 m a los $17^{\circ} 31^{\prime}$ de latitud sur y los $65^{\circ} 40^{\prime}$ de longitud oeste. La zona se caracteriza por una precipitación media anual de $530 \mathrm{~mm}$ y una temperatura media anual de $11^{\circ} \mathrm{C}$.

Se realizaron dos ensayos complementarios entre sí, uno referido a "momentos de labranza" con arado de palo y el otro referido a "implementos de labranza" con el arado de palo como testigo y considerando los mismos momentos de labranza que en el ensayo anterior. En ambos ensayos se utilizó el diseño experimental Bloques Completos al Azar con cuatro repeticiones cuyos tratamientos están descritos en las Tablas 1 y 2 respectivamente. El tamaño de cada unidad experimental en ambos ensayos fue de $10 \mathrm{~m}$ de largo por $7 \mathrm{~m}$ de ancho. Para favorecer las vueltas de la yunta durante las labranzas, las calles del ensayo tenían un ancho de $3 \mathrm{~m}$.

Los tratamientos del ensayo "Momentos de labranza" (Tabla 1) se refieren a la realización de una rayada $(R)$, arada en sentido perpendicular a la pendiente, y una cruzada (C) arada en forma paralela a la pendiente, con arado de palo, en uno, dos y/o tres momentos de labranza. Con el propósito de favorecer la preparación del suelo para siembra, en ambos ensayos en cada tratamiento se complemento con una cruzada diagonal (CD) arada en sentido diagonal a la pendiente, en el tercer momento de labranza. 
Para la cruzada diagonal se utilizó dos tipos de rastra: el bolillo de madera (tradicional) y la rastra de aletas (Foto 1 ).

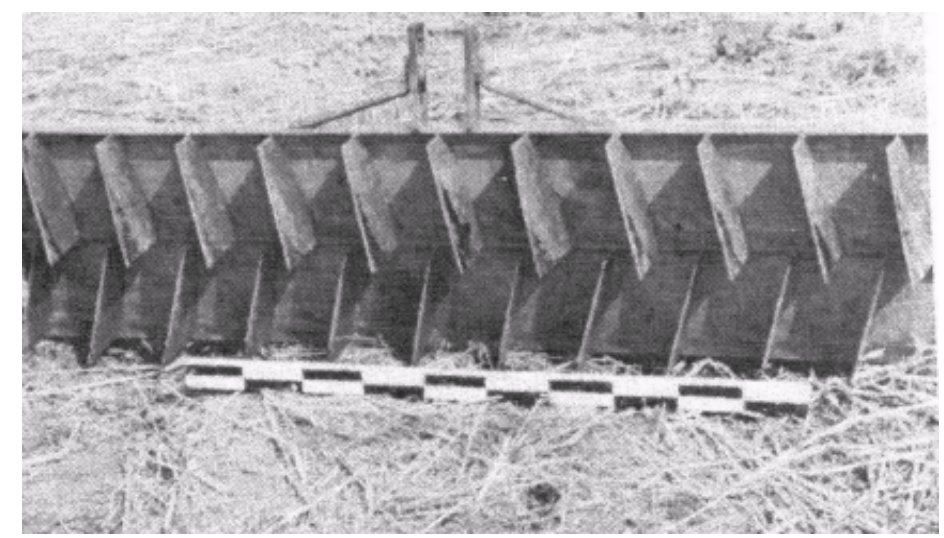

Foto 1. Rastra de aletas.

Tabla 1. Tratamientos del ensayo "momentos de labranza" con arado de palo

\begin{tabular}{|c|c|c|c|c|c|}
\hline \multirow[t]{2}{*}{ Tratamiento } & \multicolumn{5}{|c|}{ Momentos de labranza } \\
\hline & $1^{\circ}$ & $2^{-0}$ & & $3^{\circ}$ & \\
\hline L1 & & & $R+C$ & + & $C D$ \\
\hline L2 & & $\mathrm{R}+\mathrm{C}$ & & & $C D$ \\
\hline L3 & $\mathrm{R}+\mathrm{C}$ & & & & $C D$ \\
\hline L4 & & $\mathrm{R}+\mathrm{C}$ & $\mathrm{R}+\mathrm{C}$ & + & $C D$ \\
\hline L5 & $\mathrm{R}+\mathrm{C}$ & & $R+C$ & + & $C D$ \\
\hline L6 & $R+C$ & $\mathrm{R}+\mathrm{C}$ & & & $C D$ \\
\hline L7 & $R+C$ & $R+C$ & $\mathrm{R}+\mathrm{C}$ & + & $C D$ \\
\hline
\end{tabular}

$\mathrm{R}=$ Rayada; $\mathrm{C}=$ Cruzada; $\mathrm{CD}=$ Cruzada diagonal. 
Tabla 2. Tratamientos del ensayo "Implementos de labranza".

\begin{tabular}{|l|c|lll|}
\hline Tratam. & Arado & \multicolumn{3}{|c|}{ Momentos de labranza } \\
\cline { 3 - 5 } & & $\mathbf{1}^{\mathbf{0}}$ & $\mathbf{2}^{\mathbf{0}}$ & \multicolumn{1}{c|}{$\mathbf{3}^{\mathbf{0}}$} \\
\hline T1 & De palo & $\mathrm{R}$ & $\mathrm{R}+\mathrm{C}$ & $\mathrm{CD}+$ umbral \\
T2 & De palo & $\mathrm{R}$ & $\mathrm{R}+\mathrm{C}$ & $\mathrm{CD}+$ rastra de aletas \\
T3 & Reversible & $\mathrm{R}$ & $\mathrm{R}+\mathrm{C}$ & $\mathrm{CD}+$ umbral \\
T4 & Reversible & $\mathrm{R}$ & $\mathrm{R}+\mathrm{C}$ & $\mathrm{CD}+$ rastra de aletas \\
T5 & Cincel & $\mathrm{R}$ & $\mathrm{R}+\mathrm{C}$ & $\mathrm{CD}^{1}$ (A. palo) + umbral \\
T6 & Cincel & $\mathrm{R}$ & $\mathrm{R}+\mathrm{C}$ & $\mathrm{CD}^{1}$ (A. palo) + rastra de aletas \\
\hline
\end{tabular}

$\mathrm{R}=$ Rayada $; \mathrm{C}=$ Cruzada $; \mathrm{CD}=$ Cruzada diagonal.

1 Esta CD se hizo con arado de palo porque el arado cincel no remueve lo suficiente el suelo para la siembra.

Las características de los arados para tracción animal utilizados en el ensayo "Implementos de labranza" son:

- El arado de palo (Foto 2) es un implemento tradicional hecho de madera, cuyas principales partes son: mancera, telera y una reja de fierro. Requiere de humedad en el suelo para voltear la tierra (3).

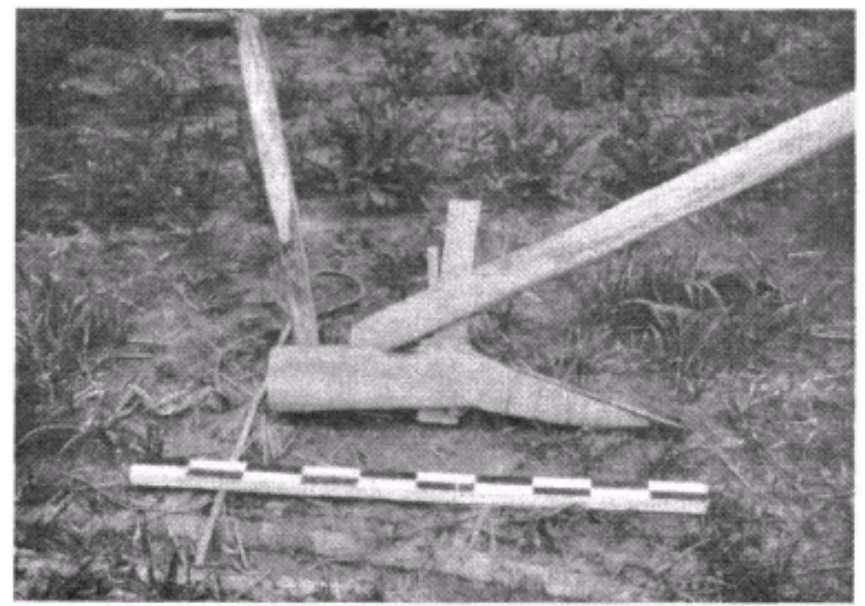


- El arado reversible (Foto 3) en un implemento hecho de fierro, cuyas principales partes son: mancera, telera, palanca reguladora de dirección de la vertedera y una vertedera hecha de acero. Requiere de humedad en el suelo para voltear la tierra.

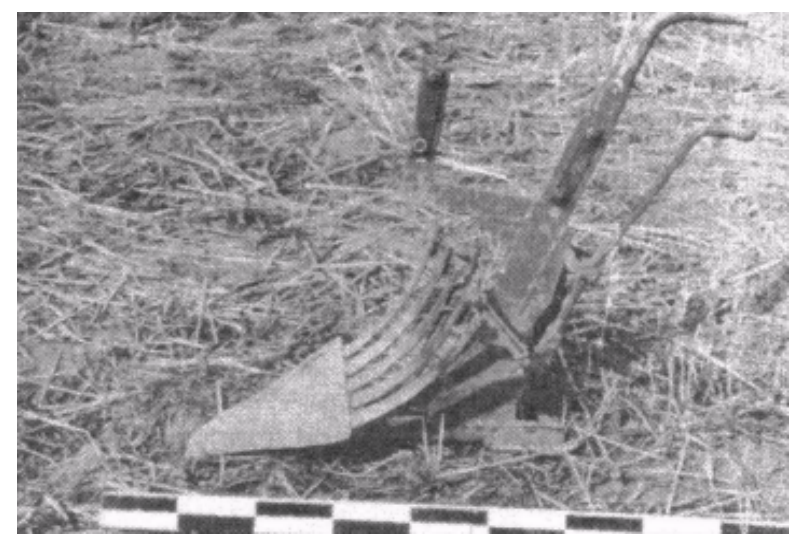

- Arado de cincel (Foto 4) en un implemento de estructura simple hecho de fierro, cuyas principales partes son: mancera, telera y una reja de acero en forma de cuchillo y diseñado para realizar fisuras en suelos secos (7).

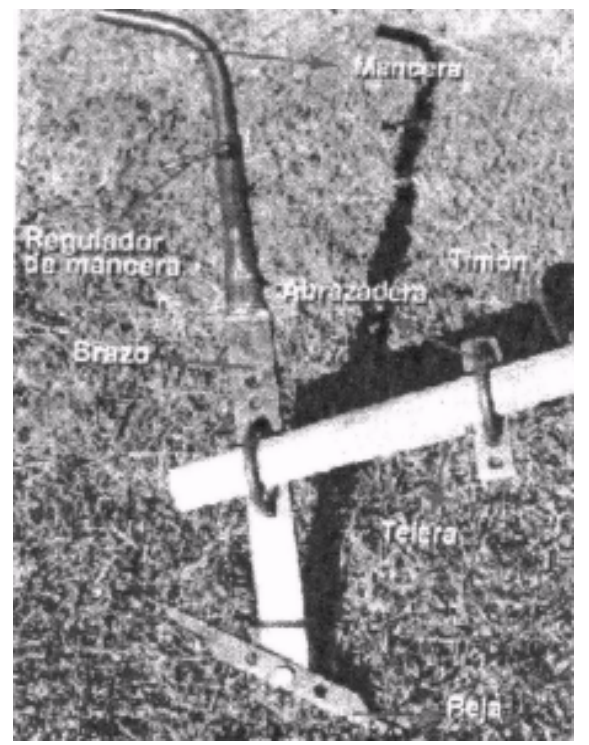


Los momentos de labranza elegidos para ambos ensayos se ajustan a épocas tradicionales de labranza en la zona andina, las cuales se realizan según la disponibilidad de humedad en el suelo. Una primera labranza se realiza aprovechando las últimas lluvias de una campaña que termina (en marzo o abril). Una segunda labranza aprovechando las nevadas ocasionales (julio o agosto) y una tercera labranza se realiza con las primeras lluvias de la campaña que se inicia (octubre). La Figura 1 describe la distribución cronológica de las labranzas de los ensayos y el momento de siembra y cosecha del cultivo. En el ensayo "implementos de labranza", por las características que tiene el arado de cincel, este se utilizó cuando el suelo se encontraba seco. Las parcelas fueron sembradas con la variedad Waych'a (ssp. andigena).

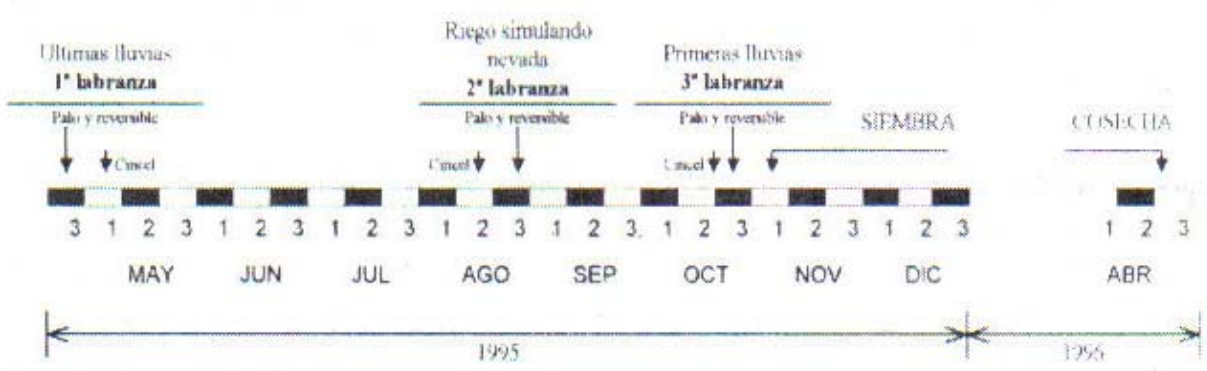

Figura 1. Descripción cronológica de las labranzas, siembra y cosecha del cultivo para ambos ensayos. Toralapa, $1995-96$

Las principales variables de suelo y del cultivo que fueron analizadas en ambos ensayos fueron:

Evaluación del suelo. Antes de las labranzas se realizaron calicatas de un metro de profundidad para evaluar las propiedades físicas y químicas de los diferentes horizontes del "perfil edafológico"1. Estas propiedades físicas y químicas se evaluaron con métodos de laboratorio. Por otra parte, en tres profundidades de la capa arable ( 0 - 10, 0 - 20 y 0 - $30 \mathrm{~cm}$ ) se determinó la densidad aparente del suelo extrayendo suelo no disturbado en anillas de volumen conocido que luego fueron secados a $110{ }^{\circ} \mathrm{C}$ y pesados. La densidad aparente junto a la densidad real del suelo que se estimó en $2.65 \mathrm{~g} / \mathrm{cc}$, permitió calcular la porosidad del suelo.

${ }^{1}$ El perfil edafológico permite identificar horizontes del suelo y sus procesos de formación a partir de la roca madre y por efecto de factores bioclimáticos (horizontes pedológicos). 
Evaluación de la humedad del suelo. Luego de las labranzas y durante el cultivo la humedad del suelo fue evaluada en forma periódica por el método gravimétrico, a tres profundidades de la capa arable $(0-10,0-20$ y $0-30 \mathrm{~cm})$ y en tres puntos de cada unidad experimental. La conductividad hidráulica del suelo fue determinada en los horizontes identificados en los "perfiles culturales" 2 (4), de acuerdo a la ley de "carga constante" de Darcy. El balance hídrico de la campaña fue estimada por el método descrito por la FAO (2).

Evaluación de la labranza. La calidad de labranza se evaluó considerando los parámetros empleados por CIFEMA (1) los cuales son: porcentaje de incorporación de rastrojo, porcentaje de desterronamiento y la superficie removida. La profundidad de aradura se determinó cuando se evaluó el "perfil cultural". La capacidad efectiva de la labranza se calculó empleando la fórmula descrita por CIFEMA (1), la cual considera básicamente el tiempo de arada y el ancho de trabajo del implemento.

Evaluación del perfil cultural. El método del perfil cultural, permitió identificar y describir los horizontes formados por las labranzas (horizontes antrópicos) con el cultivo en pleno crecimiento y permitió también determinar el efecto de las labranzas en el perfil del suelo y en el crecimiento radicular del cultivo. Esta evaluación se realizó a cuatro meses y medio de la siembra del cultivo.

Evaluación del cultivo. Las variables evaluadas en el cultivo fueron: emergencia, cobertura foliar, altura de planta, índice de cosecha y rendimiento.

\section{Resultados y Discusión}

\section{Balance hídrico}

Durante la campaña 1995 - 96 la precipitación acumulada de 625.7 $\mathrm{mm}$ fue $20 \%$ mayor respecto a la serie histórica de 13 años. El análisis del balance hídrico mostró déficit de agua durante la segunda década de noviembre y exceso durante siete décadas $(41 \%$ del ciclo). La baja permeabilidad de las capas subyacentes del suelo (a $25 \mathrm{~cm}$. de profundidad) contribuyó a incrementar el daño por exceso de agua.

${ }^{2}$ El perfil cultural permite describir la sucesión de capas de tierra formadas por la intervención de los implementos de labranza, por la acción de las raíces y por a influencia de factores climáticos (horizontes antrópicos). 


\section{Evaluación del suelo}

En la Tabla 3 se presenta una descripción resumida del perfil edafológico antes de implantar los ensayos. Las características descritas muestran que los suelos de la Estación Experimental Toralapa pertenecen al Orden Mollisoles, tal como lo describe MACA (6).

\section{Tabla 3. Descripción del perfil edafológico de un suelo de la Estación Experimental Toralapa, $(3,430 \mathrm{~m})$, Cochabamba, Bolivia, 1995 - 96}

\begin{tabular}{|c|c|c|}
\hline Hor. & Prof. (cm.) & Descripción \\
\hline $\mathrm{Ap}$ & $0-17$ & $\begin{array}{l}\text { Pardo (10YR 6/4) en seco, pardo oscuro (10YR 4/4) en } \\
\text { húmedo; textura franca; estructura granular; ligeramente } \\
\text { adhesivo y plástico; muy friable; poros frecuentes; abundantes } \\
\text { raíces finas; pH } 5.6 \text { moderadamente ácido. }\end{array}$ \\
\hline $\mathrm{A}_{12}$ & $17-29$ & $\begin{array}{l}\text { Pardo (10YR 5,5/4) en seco, pardo oscuro (10YR 4/4) en } \\
\text { húmedo; textura franca; estructura de bloques sub-angulares, } \\
\text { ligeramente plástico; friable; poros frecuentes, raíces muy } \\
\text { pocas, pH 5.75, moderadamente ácido. }\end{array}$ \\
\hline $\mathrm{Bt}$ & $29-57$ & $\begin{array}{l}\text { Pardo rojizo (5YR 4/4) en seco, pardo oscuro (5YR 3/4) en } \\
\text { húmedo; textura franco - arcillosa con gravas redondeadas de } \\
\text { naturaleza cuarcítica; estructura de bloques angulares a } \\
\text { masiva, adhesivo y plástico; muchos poros muy finos, rasgos } \\
\text { de cavidades y deyecciones de lombrices (coprolitas); muy } \\
\text { pocas raíces; pH 8.01, moderadamente alcalino. }\end{array}$ \\
\hline$\overline{C_{1}}$ & $57-87$ & $\begin{array}{l}\text { Pardo amarillento ( } 7,5 Y R \text { 5/4) en seco, pardo ( } 7,5 Y R \text { 4/4) en } \\
\text { húmedo; textura franco - arcillosa con mucha grava } \\
\text { redondeada de naturaleza cuarcítica; estructura de bloques } \\
\text { subangulares a masiva, adhesivo y plástico; muchos poros muy } \\
\text { finos, pH 8.5, fuertemente alcalino. }\end{array}$ \\
\hline $\mathrm{C}_{2}$ & $>87$ & $\begin{array}{l}\text { Pardo amarillento (10YR } 5 / 6) \text { en seco, pardo (10YR 4/4) en } \\
\text { húmedo; textura franco arcillo arenosa; con grava de naturaleza } \\
\text { cuarcítica; sin estructura; adhesivo y plástico, muy duro; } \\
\text { muchos poros continuos, pH } 8.25 \text {, fuertemente alcalino. }\end{array}$ \\
\hline
\end{tabular}

Tal como se aprecia en la Tabla 4, la densidad aparente y la porosidad del suelo antes de la labranza es similar hasta los $20 \mathrm{~cm}$. de profundidad. Las operaciones de labranza y el propio crecimiento del cultivo redujeron la densidad aparente en un 13\% y como consecuencia incrementaron la porosidad del suelo en un $17 \%$, lo que muestra que no hubo problemas de aireación. Sin embargo, hubo períodos inmediatamente después de la ocurrencia de precipitaciones que rebajaron esta aireación, lo que influyó en el desarrollo del cultivo. 
Tabla 4. Descripción de la densidad aparente y la porosidad a diferentes profundidades de un suelo de la Estación Experimental Toralapa, $(3,430 \mathrm{~m})$, Cochabamba, Bolivia, $1995-96$

\begin{tabular}{|l|lc|lc|}
\hline \multirow{2}{*}{$\begin{array}{c}\text { Capa } \\
\text { (profundidad) }\end{array}$} & \multicolumn{2}{|c|}{ Antes de la labranza } & \multicolumn{2}{c|}{ Durante el cultivo } \\
\cline { 2 - 5 } & $\mathrm{Da}\left(\mathrm{g} \mathrm{cm}^{-3}\right)$ & Porosidad (\%) & $\mathrm{Da}\left(\mathrm{g} \mathrm{cm}^{-3}\right)$ & Porosidad (\%) \\
\hline $0-10 \mathrm{~cm}$ & 1.48 & 43.7 & 1.29 & 50.9 \\
$10-20 \mathrm{~cm}$ & 1.48 & 43.7 & 1.37 & 47.9 \\
$20-30 \mathrm{~cm}$ & 1.58 & 39.9 & 1.46 & 44.5 \\
\hline
\end{tabular}

\section{Evaluación de la humedad del suelo}

De acuerdo a la Figura 2, luego de las labranzas, la humedad de la capa arable se encontraba por debajo del Punto de Marchitez Permanente (PMP). Bajo esta condición existe menor humedad en las capas superficiales respecto de las capas profundas del suelo, debido al calentamiento por la acción directa de la radiación solar que ocasionó la pérdida por evaporación. A medida que se incrementan las precipitaciones pluviales (durante el período del cultivo) y cuando éstas hacen que sobrepase la Capacidad de Campo (CC) del suelo, las capas superficiales contienen mayor humedad en relación con las capas profundas. Este efecto está ligado a la baja capacidad de estos suelos para retener agua de saturación por la presencia de capas arcillosas de permeabilidad lenta entre los 15 a $25 \mathrm{~cm}$. de profundidad.

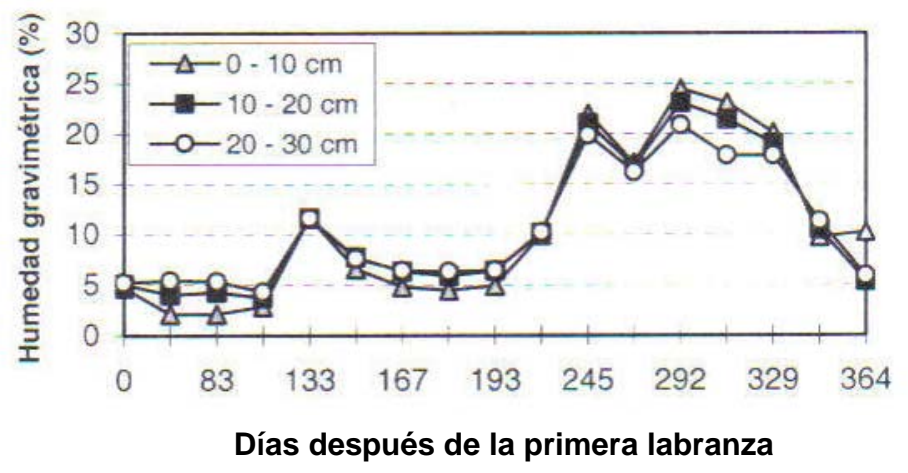

Figura 2. Evolución de la humedad a tres profundidades de un suelo de la Estación Experimental Toralapa, (3430 m), Cochabamba, Bolivia. Ensayo momentos de labranza, 1995-96. 
En la Figura 3a los tratamientos que se comparan muestran el efecto de la primera Rayada (R) y Cruzada (C) en la humedad del suelo, respecto de un suelo sin laboreo. Como se aprecia, la labranza logró conservar más agua en el suelo respecto del suelo sin labranza. De acuerdo a Orsag (8), la labranza rompe los poros capilares del suelo en los primeros $13 \mathrm{~cm}$ de profundidad, lo que actúa como una capa gruesa aislante que impide cambios bruscos de temperatura que ocasionan pérdidas de humedad en las capas inferiores.

En la Figura 3b se compara el efecto de la Rayada (R) y Cruzada (C) en la humedad del suelo, realizadas ya sea en el primer momento (L3 o L5), en el segundo momento (L2 o L4) o tanto en el primer y segundo momento (L6 o L7), respecto del testigo sin labranza (L1). $\mathrm{La}$ rayada y cruzada realizadas en el primer momento independientemente de si se realiza otra rayada y cruzada en el segundo momento, permite mantener mayor humedad en el suelo. Contrariamente, la rayada y cruzada en el segundo momento no afecta el contenido de humedad del suelo. Estos resultados muestran la importancia de la labranza inicial (primera época) aprovechando las últimas lluvias de la campaña para ayudar a conservar el agua en el suelo, lo cual puede ser importante para la producción de cultivos en años con menor precipitación. 


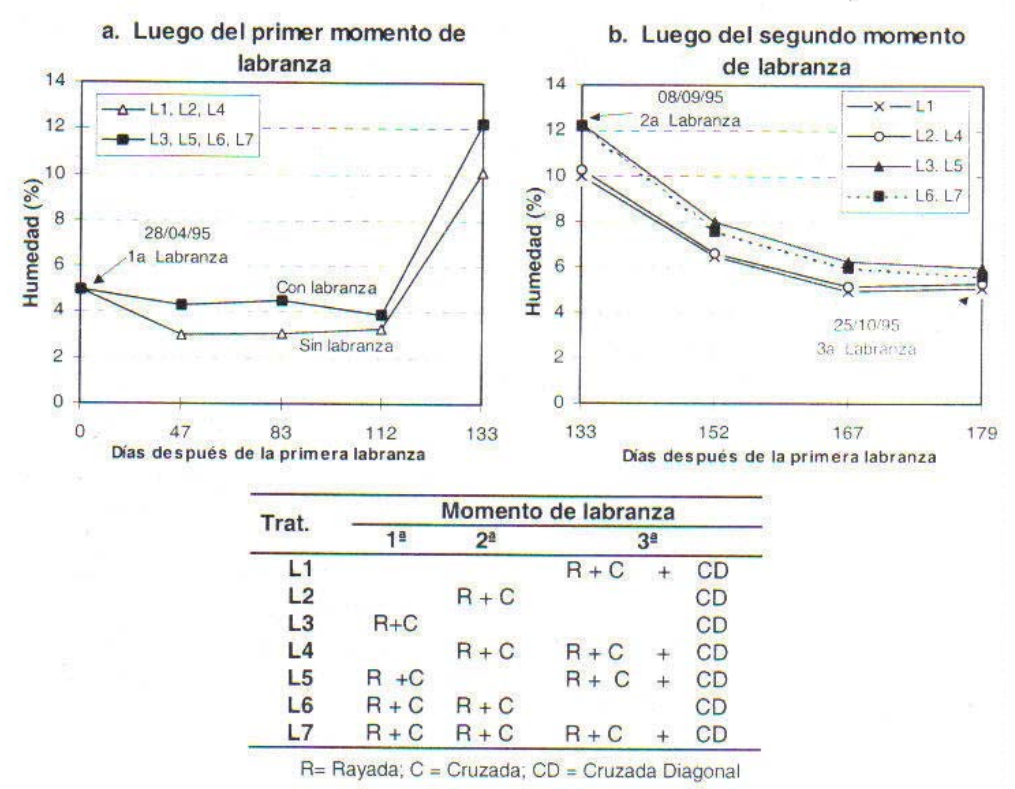

Figura 3. Variación de la humedad de un suelo de la Estación Experimental Toralapa, (3430 msnm), Cochabamba, Bolivia luego de las labranzas. Ensayo momentos de labranza, 1995-96.

En la Figura 4, donde se compara el efecto de tres implementos de labranza en el contenido de humedad en el suelo luego de la primera y segunda labranzas, se muestra que no existen diferencias marcadas entre dichos implementos, sobresaliendo el arado de cincel luego de un riego mínimo que simuló una nevada. El mayor efecto que se esperaba en la retención de humedad en el suelo por el arado de cincel no se dio, debido a que no se presentaron lluvias importantes luego de estas labranzas, cuyas aguas esperábamos podrían haber sido cosechadas por las fisuras que deja en el suelo este implemento. Durante el cultivo tampoco hubo un efecto claro del arado de cincel en la retención de agua en el suelo por que la campaña se caracterizó por ser bastante húmeda, aspecto poco común en la región donde se realizó el estudio. 
La evaluación del "perfil cultural" permitió determinar que en los horizontes antrópicos ${ }^{1}$ (de 0 a $45 \mathrm{~cm}$ de profundidad) la permeabilidad varía de moderada a lenta $\left(0.26 \mathrm{~cm} \mathrm{~h}^{-1}<k<1.44 \mathrm{~cm} \mathrm{~h}^{-1}\right)$ y en los horizontes pedológicos ( de 45 a $85 \mathrm{~cm}$ de profundidad) varía de lenta a muy lenta $\left(0.04 \mathrm{~cm} \mathrm{~h}^{-1}<\mathrm{k}<0.16 \mathrm{~cm} \mathrm{~h}^{-1}\right)$. Estos valores indican problemas de drenaje interno y coadyuvan a explicar por que el suelo tuvo elevados contenidos de humedad durante el cultivo.

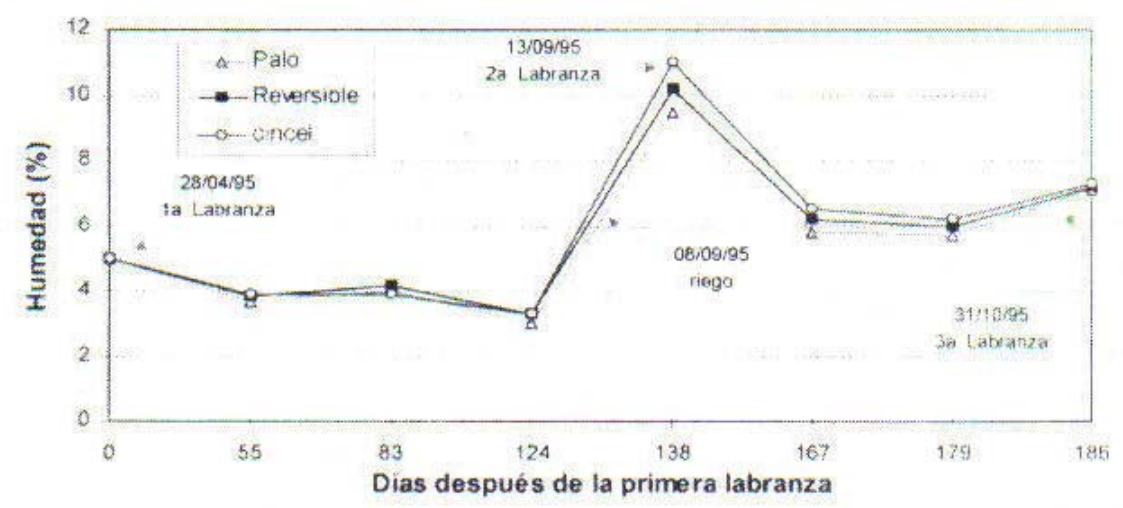

Figura 4. Variación de la humedad del suelo por efecto de tres implementos de labranza. Ensayo implementos de labranza en un suelo de la Estación Experimental Toralapa, (3,430 m), Cochabamba, Bolivia, 1995-96.

\section{Evaluación de la labranza}

En el ensayo momentos de labranza se determinó que con un número de aradas con arado de palo igual o mayor a cinco se logra incorporar el rastrojo al suelo en más de $90 \%$, lo que representa estadísticamente $9 \%$ más de rastrojo incorporado respecto a la incorporación con un número de aradas menor a cinco. El porcentaje de desterronamiento se incrementa con un mayor número de aradas y se incrementa de $70 \%$ a más de $90 \%$ cuando las aradas se realizan con más anticipación a la siembra, debido a la acción climática.

En el ensayo implementos de labranza se logró determinar que luego de cuatro aradas, el arado reversible permite un $94 \%$ de incorporación de rastrojo estadísticamente diferente al $88 \%$ de

\footnotetext{
${ }_{2}^{1}$ Horizontes formados por las labranzas y las operaciones de cultivo.

2 Agrupa a los horizontes albicos, Bt, C1 y C2, formados por procesos a partir de la roca madre y por efecto de factores bioclimáticos.
} 
incorporación por el arado de palo y al $80 \%$ por el arado de cincel. No se pudo demostrar que existan diferencias entre los implementos de labranza en el porcentaje de desterronamiento. Ajustando el ancho de trabajo (distancia entre una y otra arada) a $0.5 \mathrm{~m}$ para todos los implementos estudiados, se determino un porcentaje de superficie removida de $83 \%, 57 \%$ y $38 \%$ para el arado reversible, de palo y de cincel respectivamente.

La comparación de las rastras muestra que el umbral (bolillo de madera) hinca los terrones más duros y alisa la superficie aparentando mayor desterronamiento; en cambio la rastra de aletas, penetra $10 \mathrm{~cm}$ con las aletas y corta los terrones grandes.

En la Tabla 5 se presentan algunas variables que muestran la efectividad de los implementos en las operaciones de laboreo. Tanto con el arado de palo como con el arado reversible, la cruzada diagonal demandó aproximadamente un $16 \%$ más de tiempo respecto a la rayada y cruzada. De acuerdo a la capacidad efectiva (CE), que considera básicamente el tiempo de arada y el ancho de trabajo del implemento, la rayada y cruzada con arado de cincel demandan $38 \%$ más de tiempo que el arado de palo y $63 \%$ más de tiempo que el arado reversible para trabajar una hectárea. Esto se debe al menor ancho de labor que realiza el arado de cincel y no así a su velocidad de laboreo que es mayor al del arado reversible.

Tabla 5. Ancho de trabajo, velocidad de laboreo y capacidad efectiva (CE) de los implementos estudiados, según la operación realizada.

\begin{tabular}{|c|c|c|c|c|c|c|}
\hline \multirow[t]{2}{*}{ Implemento } & \multirow{2}{*}{$\begin{array}{c}\text { Ancho } \\
\text { trabajo } \\
{[\mathrm{m}]}\end{array}$} & \multirow{2}{*}{$\begin{array}{l}\text { Velocidad } \\
{\left[\mathrm{m} \mathrm{s}^{-1}\right]}\end{array}$} & \multirow{2}{*}{$\begin{array}{l}\text { Pérd. por } \\
\text { vuelta [s] }\end{array}$} & \multirow{2}{*}{ Operación } & \multicolumn{2}{|c|}{ CE } \\
\hline & & & & & {$\left[\mathrm{h} \mathrm{ha}{ }^{-1}\right]$} & $\begin{array}{l}{[\mathrm{ha}} \\
\left.\mathrm{h}^{-1}\right]\end{array}$ \\
\hline Arado de palo & 0.32 & 0.95 & 8.38 & $\begin{array}{c}\text { Rayada } \\
\text { Cruzada } \\
\text { Cruzada diagonal }\end{array}$ & $\begin{array}{c}11.2 \\
11.2 \\
13\end{array}$ & $\begin{array}{l}0.089 \\
0.089 \\
0.077\end{array}$ \\
\hline Arado reversible & 0.42 & 0.86 & 11.08 & $\begin{array}{c}\text { Rayada } \\
\text { Cruzada } \\
\text { Cruzada diagonal }\end{array}$ & $\begin{array}{l}9.5 \\
9.5 \\
11\end{array}$ & $\begin{array}{l}0.105 \\
0.105 \\
0.091\end{array}$ \\
\hline Arado cincel & 0.23 & 0.96 & 8.95 & $\begin{array}{l}\text { Rayada } \\
\text { Cruzada }\end{array}$ & $\begin{array}{l}15.5 \\
15.5\end{array}$ & $\begin{array}{l}0.064 \\
0.064\end{array}$ \\
\hline Umbral & 1.43 & 0.83 & 13 & Rastrada & 2.9 & 0.345 \\
\hline Rastra de aletas & 1.43 & 0.99 & 13 & Rastrada & 2.5 & 0.4 \\
\hline
\end{tabular}




\section{Evaluación del perfil cultural}

En la evaluación del perfil cultural se constató la formación de cuatro horizontes antrópicos formados por las operaciones del cultivo y las labranzas: el primero próximo al cuello de las plantas formado por los aporques $(\mathrm{H} 1)$; el segundo formado por el surcado $(\mathrm{H} 2)$ y que tiene una profundidad máxima de $13 \mathrm{~cm}$; el tercero formado por las labranzas (H3) cuyo límite es el "fondo de arado" o el "pie de arado", según sea el implemento usado.

En la Tabla 6 se muestra el efecto del número de aradas y del tipo de arado, en la profundidad de aradura. A un mayor número de aradas la profundidad de aradura es mayor, llegando a tocar el pie de arado cuando el número de aradas es mayor a siete. Por otra parte, el arado de cincel permite una mayor profundidad de aradura respecto al arado reversible y al arado de palo, llegando a raspar el pie de arado y a romperlo en algunos sectores.

Tabla 6. Profundidad de arada según el tipo de arado y el número de aradas.

\begin{tabular}{|c|c|c|c|c|c|}
\hline Ensayo & Trat. & Arado & $\mathrm{N}^{\circ}$ Aradas & $\begin{array}{l}\text { Prof. } \\
{[\mathrm{cm} .]}\end{array}$ & Observaciones \\
\hline $\begin{array}{l}\text { Momentos } \\
\text { de labranza }\end{array}$ & $\begin{array}{l}\text { L1 } \\
\text { L5 } \\
\text { L7 }\end{array}$ & Palo & $\begin{array}{l}3 \\
5 \\
7\end{array}$ & $\begin{array}{l}7-13 \\
18-20 \\
20-22\end{array}$ & Toca pié de arado \\
\hline Implementos & T2 & Palo & 4 & $10-17$ & \\
\hline de labranza & $\begin{array}{l}\text { T4 } \\
\text { T5 }\end{array}$ & Reversible & 3 & $\begin{array}{l}20-23 \\
22-28\end{array}$ & $\begin{array}{l}\text { Toca pié de arado, lo } \\
\text { raspa en un sector } \\
\text { Raspa el pié de arado, } \\
\text { lo rompe en un sector }\end{array}$ \\
\hline
\end{tabular}

La Figura 5 es un esquema del perfil cultural donde se compara el efecto del arado de cincel con el arado de palo, se observa que el arado de cincel logra romper el pie de arado en algunos tramos. También se observa que en la superficie no se formó costra alguna debido a la textura franca del suelo; y el límite de cada horizonte antrópico es una costra formada por hidromorfía con espesores entre 0.5 y $2 \mathrm{~cm}$. e incluso hasta $4 \mathrm{~cm}$. en el pié de arado. Estas costras son limitantes para el arraigamiento y el movimiento del agua por su compactación y estructura laminar. 

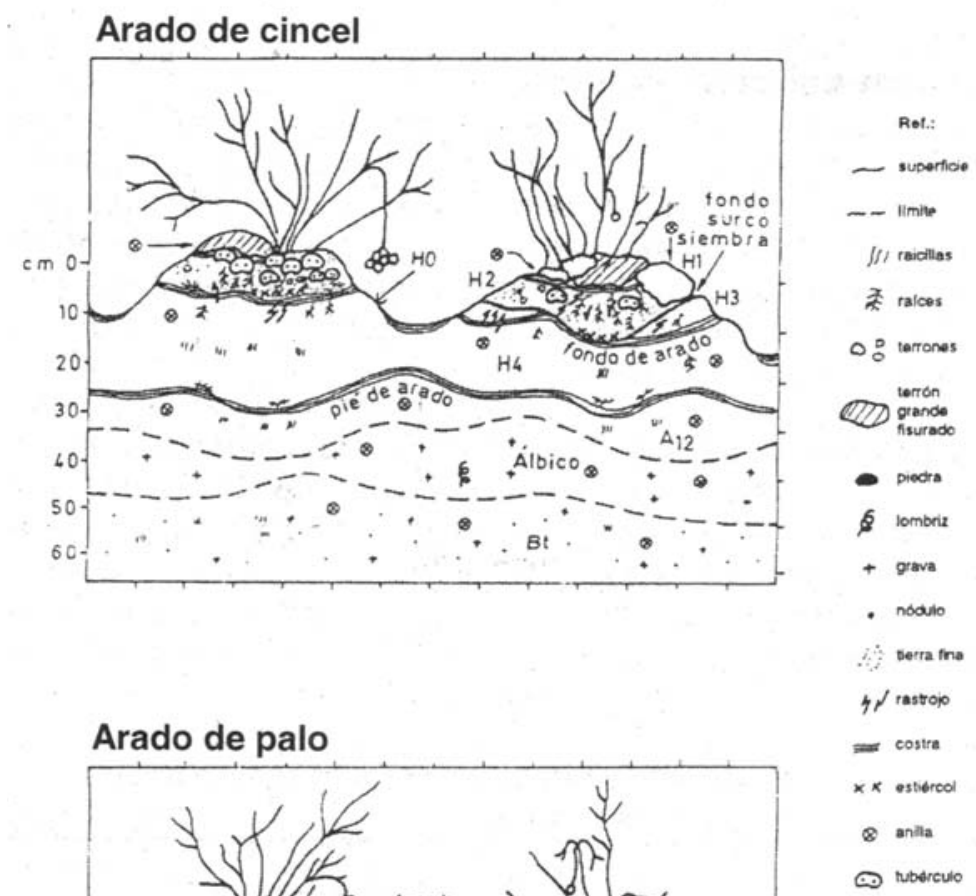

Figura 5.

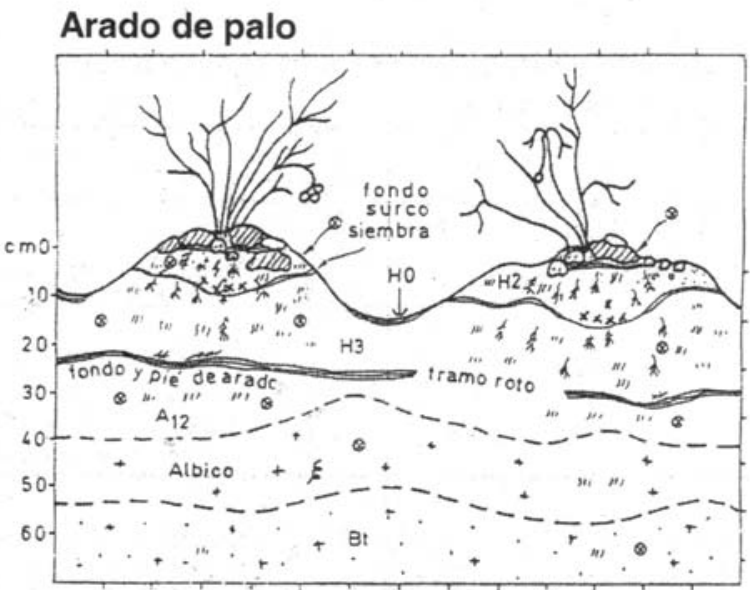

\begin{abstract}
Toralapa, $(3,430 \mathrm{~m})$, Cochabamba, Bolivia, con tres aradas con arado cincel y con arado de palo. Ensayo implementos de labranza, 1995-96.
\end{abstract}

El $70-80 \%$ del enraizamiento se produjo hasta el fondo del surco de siembra y un 25 a $15 \%$ entre éste y el fondo de arado. Se destaca el suelo arado con cincel donde el enraizamiento es más uniforme con 55 a $60 \%$ hasta el fondo del surco de siembra y 40 a 35\% entre éste y el fondo de arado. Se observó raicillas de papa hasta $70 \mathrm{~cm}$ de profundidad, debido a la porosidad de los horizontes pedológicos y a la actividad de las lombrices indicada por la existencia de canalículos por donde circulan, cavidades donde se alojan y deyecciones 
(coprolitas). La tuberización se produjo en todos los casos entre el horizonte formado por la siembra y el formado por los aporques, es decir, en el camellón del surco.

\section{Evaluación del cultivo}

La emergencia, la cobertura foliar y la altura de planta no fueron afectadas significativamente por los tratamientos de ambos ensayos. Respecto al índice de cosecha se constató, en ambos ensayos, que tiene relación con la profundidad de arada y el enraizamiento porque en el ensayo épocas de labranza el mayor índice corresponde al tratamiento con siete aradas (L7) con $79 \%$, aunque respecto a los otros no es significativo.

En el ensayo implementos de labranza, el mejor índice de cosecha fue observado para la combinación del arado cincel con la rastra de aletas (T6) con $84 \%$ seguido del tratamiento con arado reversible y rastra de aletas (T4) con 82\%; y el menor índice es del tratamiento con arado de palo y umbral (T1) con $73.4 \%$. Existieron diferencias estadísticas sólo entre el T6 y el T1.

Como se observa en la Tabla 7 y tal como ocurrió con las variables de crecimiento evaluadas, no se encontraron diferencias estadísticas en el rendimiento del cultivo de papa por efecto del momento en que se realizó la rayada $(R)$ y cruzada $(C)$ con el arado de palo y/o el número de veces en que se realizaron estas operaciones (de 1 a 3 veces). Estos resultados muestran que un excesivo laboreo no repercute en un incremento del rendimiento de papa bajo las condiciones del ensayo.

Por tanto, considerando que una rayada y una cruzada son suficientes para incrementar el rendimiento respecto a un suelo sin laboreo, para una posible recomendación se descartan todos aquellos tratamientos que involucran más de una rayada y cruzada como es el caso de los tratamientos L4, L5, L6 Y L7. La decisión se debería tomar entre los tratamientos L1, L2 y L3, pero la aleatoria ocurrencia de nevadas en el mes de agosto, descarta a la segunda época de labranza como una opción de recomendación, por tanto con este criterio L2 también quedaría descartada, quedando sólo L1 y L3. Para elegir una u otra alternativa, el agricultor debería considerar la disponibilidad de mano de obra en una y otra época, y también la mayor capacidad de retención de agua que se logra cuando se ara el suelo en la primera época (Figura $2 b$ ), aspecto que es de importancia sobre todo en años de baja precipitación. 
Tabla 7. Rendimiento total y por categoría sobre la base del tamaño de tubérculos en el ensayo momentos de labranza. Estación Experimental Toralapa, (3,430 m), Cochabamba, Bolivia, 1995-96.

\begin{tabular}{|c|c|c|c|c|c|c|c|c|c|c|}
\hline \multirow[t]{2}{*}{ Trat. } & \multicolumn{4}{|c|}{ Momento de labranza* } & \multicolumn{6}{|c|}{ Rendimiento $\left|\mathrm{t} \mathrm{ha}{ }^{-1}\right|^{* *}$} \\
\hline & \multicolumn{4}{|c|}{$\begin{array}{lll}1^{\mathrm{a}} & 2^{\underline{a}} & 3^{a^{a}}\end{array}$} & I & II & III & IV & \multicolumn{2}{|c|}{ Total } \\
\hline L1 & & & $R+C$ & + & 1.05 & 4.56 & 6.49 & 6.05 & 18.55 & $A$ \\
\hline L2 & & $\mathrm{R}+\mathrm{C}$ & & & 0.26 & & 6.23 & 5.69 & & $A B$ \\
\hline L3 & $R+C$ & & & c & 0.52 & 4.0 & 7.64 & 6.51 & & A \\
\hline L4 & & $R+C$ & $R+C$ & + & 0.93 & 3.56 & 7.46 & 5.24 & 17.19 & A \\
\hline L5 & $R+C$ & & $R+C$ & + & 1.07 & 3.63 & 7.48 & 5.44 & 17.62 & A \\
\hline 10 & & $\mathrm{R}+\mathrm{C}$ & & C & 0.20 & & & 6.4 & & $A B$ \\
\hline & & $R+C$ & $R+C$ & + & 0.42 & 2.47 & 6.88 & 6.92 & 16.69 & $A B$ \\
\hline \multicolumn{5}{|c|}{ CV \% } & 93.57 & 38.45 & 20.67 & 14.71 & 17.06 & \\
\hline
\end{tabular}

* $\mathrm{R}=$ Rayada; Cruzada; $\mathrm{CD}=$ Cruzada diagonal.

** Tamaño de tubérculos: I = > a $5 \mathrm{~cm}$; II $=4.5$ a $5 \mathrm{~cm}$; III $=3.5$ a $4.5 \mathrm{~cm}$; IV = $<$ a $3.5 \mathrm{~cm}$.

Como se observa en la Tabla 8, todos los tratamientos que incluyen la rastra de aletas tienden a incrementar el rendimiento del cultivo de papa. Estadísticamente cuando se combina el arado reversible con la rastra de aletas (T4) el efecto en el rendimiento es mayor que cuando se combina con umbral (T3). Este efecto se debe a que el arado reversible al voltear más tierra logra exponer más terrones a la superficie los cuales son mullidos de mejor manera por la rastra de aletas que por el umbral (bolillo de madera), el cual sólo alisa la superficie aparentando más desterronamiento.

Una tendencia similar se observa en la combinación del arado de cincel con la rastra de aletas, pero el incremento de rendimiento no fue significativo respecto a la combinación con el umbral. 
Tabla 8. Rendimiento total y por categoría sobre la base de tamaño de tubérculos en el ensayo implementos de labranza. Estación Experimental Toralapa, (3,430 m), Cochabamba, Bolivia, 199596.

\begin{tabular}{|c|c|c|c|c|c|c|c|c|c|}
\hline \multirow[b]{2}{*}{ Trat. } & \multirow[b]{2}{*}{ Arado } & \multicolumn{3}{|c|}{ Momento de labranza* } & \multicolumn{5}{|c|}{ Rendimiento $\left[\mathrm{t} \mathrm{ha}^{-1}\right]^{\star \star}$} \\
\hline & & $1^{\underline{a}}$ & $2^{\underline{a}}$ & $3^{\underline{a}}$ & $\mathrm{I}$ & II & III & IV & Total \\
\hline T1 & Palo & $\mathrm{R}$ & $R+C$ & CD + umbral & 0 & 1.31 & 4.90 & 6.03 & 1224 \\
\hline T2 & Palo & $\mathrm{R}$ & $R+C$ & $\mathrm{CD}+$ rastra de aletas & 0 & 1.55 & 5.42 & 7.26 & $14.23 \mathrm{AB}$ \\
\hline T3 & Rev. & $\mathrm{R}$ & $\mathrm{R}+\mathrm{C}$ & $C D+$ umbral & 0 & 0.10 & 4.13 & 6.79 & $1102 \quad B$ \\
\hline T4 & Rev. & $\mathrm{R}$ & $\mathrm{R}+\mathrm{C}$ & $C D+$ rastra de aletas & 0.89 & 3.65 & 8.06 & 7.02 & $19.62 \mathrm{~A}$ \\
\hline T5 & Cincel & $\mathrm{R}$ & $\mathrm{R}+\mathrm{C}$ & $C D$ (A. palo) + umbral & 0.20 & 2.14 & 5.91 & 5.67 & $13.93 \mathrm{AB}$ \\
\hline T6 & Cincel & $\mathrm{R}$ & $\mathrm{R}+\mathrm{C}$ & $\begin{array}{l}C D(A . \text { palo })+\text { rastra de } \\
\text { aletas }\end{array}$ & 057 & 2.90 & 6.75 & 6.01 & $16.23 \mathrm{AB}$ \\
\hline & & & & $\mathrm{CV} \%$ & 172.3 & 110.17 & 37.17 & 22.05 & 28.89 \\
\hline
\end{tabular}

${ }^{*} \mathrm{R}=$ Rayada; $\mathrm{C}=$ Cruzada; $\mathrm{CD}=$ Cruzada diagonal.

**Tamaño de tubérculo: I = > a $5 \mathrm{~cm}$.; II = 4.5 a $5 \mathrm{~cm}$.; III = 3.5 a $4.5 \mathrm{~cm}$; IV = < a 3.5 $\mathrm{cm}$.

\section{Conclusiones}

- Los suelos de la Estación Experimental Toralapa hasta los $30 \mathrm{~cm}$. de profundidad son de textura $F$, de una estructura que varia de granular a bloques angulares y de un $\mathrm{pH}$ moderadamente ácido. Entre los 30 a $85 \mathrm{~cm}$. de profundidad son de textura FY, estructura de bloques angulares y de $\mathrm{pH}$ moderadamente alcalino a fuertemente alcalino. A una profundidad mayor a $85 \mathrm{~cm} \mathrm{la}$ textura es FYA, sin estructura y de $\mathrm{pH}$ fuertemente alcalino.

- Cuando la humedad del suelo se encuentra por debajo de la humedad aprovechable por el cultivo, las capas inferiores (20 a $30 \mathrm{~cm}$. de profundidad) tienen más humedad que las capas superficiales (0 - $10 \mathrm{~cm}$. de profundidad) y ocurre un caso inverso cuando la humedad del suelo se encuentra por encima de la capacidad de campo del suelo.

- La labranza permite conservar más agua en el suelo respecto de un suelo sin labranza, debido a que ésta logra romper los microporos de la capa arable, actuando así como una capa aislante que impide los cambios bruscos de temperatura que ocasionan las pérdidas de humedad de las capas inferiores.

- Las condiciones de alta humedad de la campaña (20 \% más respecto al promedio histórico) no permitió demostrar el efecto del arado de cincel en la retención de humedad en el suelo y la cosecha de agua mediante la apertura de fisuras en suelo seco 
rompiendo las capas duras. Este trabajo no se puede realizar con el arado de palo.

- El porcentaje de desterronamiento se incrementa cuando las aradas se realizan con más anticipación a la siembra, debido a que las condiciones climáticas más húmedas favorecen este efecto.

- El arado reversible logra una mayor incorporación de rastrojo y logra una mayor superficie removida respecto al arado de palo y al arado de cincel.

- La rayada y cruzada con arado de cincel demanda más tiempo respecto al arado de palo y al arado reversible.

- A un mayor número de aradas con arado de palo la profundidad de aradura es mayor, llegando a tocar el pie de arado cuando el número de aradas es mayor a siete.

- El arado de cincel permite una mayor profundidad de aradura respecto al arado reversible y al arado de palo, llegando a raspar el pie de arado y a romperlo en algunos sectores, permitiendo así un enraizamiento más uniforme.

- No se pudo demostrar el efecto de las épocas de labranza y del número de aradas sobre el rendimiento del cultivo de papa. Por tanto, desde el punto de vista agronómico y de conservación del suelo, el número de aradas debería ser menor a tres y estas realizadas en una sola época de preferencia al final de la campaña anterior para aprovechar mejor la humedad remanente del suelo.

- No se pudo demostrar diferencias en el rendimiento por efecto de los implementos evaluados excepto con el uso de rastra de aletas frente al umbral. Por las bondades del arado de cincel en la ruptura del pie de arado y en el crecimiento de las raíces del cultivo, es probable que su efecto en el rendimiento fue enmascarado por la excesiva humedad de la campaña.

\section{Referencias Bibliográficas}

1. CIFEMA. 1990. Evaluación comparativa de arados de vertedera reversible para tracción animal. En: Revista de Agricultura (Año 47, Mayo 1990, No. 16). Cochabamba, Bol., Facultad de Ciencias Agrícolas y Pecuarias, p. 2-13. 
2. FAO; SNAG; FERTISUELOS. 1994. Guía metodológica de descripción rápida de los perfiles culturales para la identificación de las limitaciones al arraigamiento y el establecimiento de balances hídricos. GCPE / BOL / 018 / NET. Cochabamba, Bol. 9 $P$.

3. Flores, D.; Altamirano, W.; Cárdenas, J. 1996. Análisis comparativo de herramientas tradicionales de labranza en el departamento de Oruro. Editado por Herve, Condori y Orsag en Las labranzas en perspectiva. La Paz, Bolivia, IBTA - ORSTOM, Informe ORSTOM No 52, pp 9 a 17.

4. Gautronneau Y.; Manichon, H.; Herve, D.; Ramos, D. 1996. Guía metódica del perfil cultural. La Paz, IBTA - ORSTOM, Informe No. $54,27 \mathrm{p}$.

5. Herve, D.; Condori, D.; Orsag, V. 1996. Las labranzas en perspectiva. Andes Centrales. La Paz, IBTA - ORSTOM, Informe ORSTOM No. 52, 114 p.

6. MACA. 1977. Clasificación taxonómica y de capacidad de uso de las tierras productoras de papa. Tiraque - Toralapa - Epizana. 96 P.

7. Mamani P.; Botello, R.; Condori, R.; Devaux, A. 1999. Labranza en suelos secos para la cosecha de aguas. El arado de cincel. Ficha Técnica No. 1, Sistemas de Producción, Fundación PROINPA.

8. Orsag, V. 1996. Evaluación de la disponibilidad de agua en el suelo franco arcilloso arenoso del Altiplano norte. Editado por Herve, Condori y Orsag en Las labranzas en Perspectiva. La Paz, Bolivia, IBTA - ORSTOM, Informe ORSTOM No. 52:57-65.

9. PROINPA. 1998. Informe Compendio del Programa de Investigación de la Papa. Cochabamba, Bolivia. 62 p. 